\title{
A COMPLETE AND COUNTABLE ORTHOMODULAR LATTICE IS ATOMIC
}

CHARLES H. RANDALL

To prove the assertion in the title, let $\left(L ; \leqq,^{\prime}\right)$ be a countable orthomodular lattice. If $L$ is not atomic, there exists a nonzero $b \in L$ that does not majorize an atom. In $L$, let $f<g \leqq b$. By the orthomodular identity $g=f \vee\left(f^{\prime} \wedge g\right)$ and necessarily $\left(f^{\prime} \wedge g\right) \neq 0$. Since $\left(f^{\prime} \wedge g\right) \leqq g \leqq b,\left(f^{\prime} \wedge g\right)$ can not be an atom and there exists in $L$ a nonzero $d<\left(f^{\prime} \wedge g\right)$. Again $\left(f^{\prime} \wedge g\right)=d \vee\left(d^{\prime} \wedge f^{\prime} \wedge g\right)$ and $\left(d^{\prime} \wedge f^{\prime} \wedge g\right)$ $\neq 0$. Let $h=f \vee d$. Since $g=f \vee d \vee\left(d^{\prime} \wedge f^{\prime} \wedge g\right)$ is an orthogonal join of nonzero elements, it follows that $f<h<g \leqq b$. Thus the interval $[0, b]=\{g \mid 0 \leqq g \leqq b\}$ is order dense-in-itself (i.e., if $f<g$ in $[0, b]$ there exists an $h \in[0, b]$ such that $f<h<g)$.

Let $C$, by Zorn's Lemma, be a maximal chain in $[0, b]$. It follows that $C$ is bounded, countable and order dense-in-itself. Hence $C$ must be order isomorphic to the chain of all rational numbers between any pair of fixed distinct endpoints (see [1, p. 200]). It is well known that such a chain is not order complete. Therefore there exists a $C^{\prime} \subseteq C$ for which no $\mathrm{V} C^{\prime}$ exists in $C$. If such an element $\mathrm{V} C^{\prime}$ exists in $L$, it must be in $[0, b]$, for $b$ is an upper bound of $C^{\prime}$. In that event, by the maximality of $C, V C^{\prime}$ must also be in $C$. Thus no $V C^{\prime}$ exists in $L$ and $L$ is not complete. Q.E.D.

\section{REFERENCE}

1. Garrett Birkhoff, Lattice theory, 3rd ed., Amer. Math. Soc. Colloq. Publ., Vol. 25, Amer. Math. Soc., Providence, R. I., 1967.

UNIVERSITY OF MASSACHUSETTS

Received by the editors April 3, 1968. 